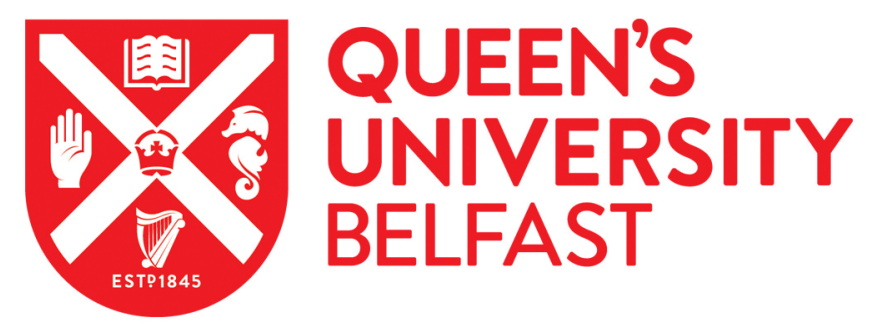

\title{
Similarities and Differences in Health-Related Quality of Life Concepts Between the East and the West: A Qualitative Analysis of the Content of Health-Related Quality of Life Measures
}

Mao, Z., Ahmed, S., Graham, C., Kind, P., Sun, Y-N., \& Yu, C-H. (2021). Similarities and Differences in HealthRelated Quality of Life Concepts Between the East and the West: A Qualitative Analysis of the Content of Health-Related Quality of Life Measures. Value in Health Regional Issues, 24, 96-106.

https://doi.org/10.1016/j.vhri.2020.11.007

Published in:

Value in Health Regional Issues

Document Version:

Peer reviewed version

Queen's University Belfast - Research Portal:

Link to publication record in Queen's University Belfast Research Portal

\section{Publisher rights}

Copyright 2021 Elsevier Inc.

This manuscript is distributed under a Creative Commons Attribution-NonCommercial-NoDerivs License

(https://creativecommons.org/licenses/by-nc-nd/4.0/), which permits distribution and reproduction for non-commercial purposes, provided the author and source are cited.

\section{General rights}

Copyright for the publications made accessible via the Queen's University Belfast Research Portal is retained by the author(s) and / or other copyright owners and it is a condition of accessing these publications that users recognise and abide by the legal requirements associated with these rights.

\section{Take down policy}

The Research Portal is Queen's institutional repository that provides access to Queen's research output. Every effort has been made to ensure that content in the Research Portal does not infringe any person's rights, or applicable UK laws. If you discover content in the

Research Portal that you believe breaches copyright or violates any law, please contact openaccess@qub.ac.uk. 
Similarities and Differences in HRQoL concepts between the East and the West - a qualitative analysis of the content of HRQoL measures

Authors: Zhuxin Mao ${ }^{1}$, Shenaz, Ahmed ${ }^{1}$, Christopher Graham², Paul Kind ${ }^{1}$, Ya-Nan Sun $^{3}$, Chang-He $\mathrm{Yu}^{4 *}$

1. Institute of Health Sciences, University of Leeds, Leeds, England;

2. Department of Psychology, Queens' University Belfast, Belfast, England;

3. Department of Traditional Chinese Medicine, Xuanwu Hospital Capital Medical University, Beijing, China;

4. Tuina and Pain Management Department, Dongzhimen Hospital, Beijing University of Chinese Medicine, Beijing, China

*Corresponding author: Chang-He Yu, yakno2@163.com, 86-18601159559 


\section{Abstracts:}

\section{Background}

Most of the commonly used HRQoL measures were developed in the West and have been introduced into other countries for use worldwide. Arguably, this adaption process assumes that health, as a concept, has universal cultural equivalence. This study identified those Chinese-developed HRQoL measures and summarised their contents, with which the Western-developed HRQoL measures were compared, aiming to explore cultural differences in defining and measuring health between the East and the West.

\section{Methods}

A systematic literature search was conducted to identify Chinese-developed generic HRQoL measures. Two Western-developed HRQoL measures (EQ-5D, SF-36) and a cross-culturally developed measure (WHOQOL-100) were included for comparison. A qualitative content analysis was undertaken both deductively and inductively to categorise and summarise the content of the questionnaires in analysing similarities and differences between Western and Chinese-developed HRQoL measures.

\section{Results}

Eight HRQoL measures that were designed in a Chinese cultural context were identified and compared with the three non-Chinese-developed HRQoL measures. Although there is an agreement between the East and the West regarding the domains and subdomains of HRQoL, health concepts including "emotion control”, "weather 
adaption”, “social adaption”, “spirit”, and “complexion” were exclusively introduced by the Chinese measures.

\section{Conclusions}

The results demonstrate that health is a culturally grounded concept and, due to cultural differences, it cannot be taken for granted that a well recognised Western HRQoL measure is always appropriate for use in other cultural contexts. This study implies the necessity of further examining the legitimacy of applying Westerndeveloped HRQoL measures in other cultural settings. 


\section{Highlights:}

- Most of the commonly used generic HRQoL measures were developed in Europe or North America and have been translated into other languages for use worldwide. Arguably, this adaption process assumes that health, as a concept, has universal cultural equivalence.

- This paper aims to explore cultural differences in defining and measuring health to argue that Western-developed HRQoL measures might not be appropriate for use in China, whose culture is considered to be significantly different from that in the West.

- The results demonstrate that health is a culturally grounded concept and, due to cultural differences, it cannot be taken for granted that a well recognised Western HRQoL measure is always appropriate for use in other cultural contexts. Careful consideration and testing of conceptual equivalence is essential when deciding whether to use an existing HRQoL measure outside of the culture in which it was developed. 


\section{Background:}

With a significant decrease in mortality and the trend of treating chronic diseases as major health problems, health professionals, policymakers and health care providers has developed an increasing concern over people's satisfaction and feeling, instead of merely looking at traditional outcome indicators, such as physical signs and conventional clinical indexes [1-3]. Health-related quality of life (HRQoL), which can be understood as a subjective, multidimensional concept that is associated with individuals' self-perception of their own health, has become an important component of health outcomes [4,5]. Although defining HRQoL has been challenging [4,6,7], it is widely agreed that this concept is with a self-perceived" perspective, focusing on revealing people's subjective evaluation of health [8], while most definitions of HRQoL incorporate dimensions of physical function, mental/psychological wellbeing, social function, role function and global perceptions of function and well-being $[4,9,10]$.

HRQoL is often used interchangeably with terms like health status and quality of life (QoL) [4,11]. There is a general lack of consensus over the definitions and measurement of these concepts [12]. As Karimi and Brazier illustrated, measures such as EQ-5D and SF-36 are referred to as "health status measures” [13,14], "HRQoL measures” $[15,16]$ and “QoL measures” $[17,18]$ in different papers. In this paper, the term “HRQoL measures” was chosen to represent such health measures to reflect their "self-perceived” and "health-focused” features. 
Most of the commonly used HRQoL measures, such as EQ-5D and SF-36, were developed in Europe or North America [19]. Widely-used English language questionnaires are often translated into other languages and for worldwide use [20,21]. Translating available HRQoL measures can facilitate researchers in nonEnglish speaking countries to conduct HRQoL studies. Another reason for introducing HRQoL to other countries or cultures may be that researchers are interested in doing cross-country/culture comparative studies by using the same HRQoL measure with different languages versions [22]. Additionally, researchers tend to trust and apply those HRQoL measures that are supported by accumulating evidence of validity and have already been used widely.

However, translating HRQoL measures into other countries or cultures may be problematic $[19,20,23,24]$. Health is a culturally relevant concept $[25,26]$ and therefore, health measures that were developed in a Western cultural setting may contain concepts that are not necessarily relevant or important in other cultures [27]. Previous studies have shown that Chinese people have different views about health compared to Westerners [28-31]. For example, several studies have found that Chinese people used concepts such as spirits, food and weather, which are less likely to be referred to by people in Western countries, in describing health [28, 32, 35]. The legitimacy of applying Western-developed HRQoL measures in China has also been questioned by empirical findings [33,34]. For example, a study reported insufficient internal consistency and unsatisfactory item-to-scale correlation in Chinese participants when SF-36 was used [17]. Generic HRQoL measures reflecting Chinese 
culture were accordingly developed, with the aim of better-describing health status among Chinese people [35].

Several studies reviewed applications of HRQoL measures in China, but they focused on those measures developed from a Traditional Chinese Medicine (TCM) perspective $[36,37]$. Other studies have reviewed the used of Western HRQoL measures, such as EQ-5D and SF-6D, in Chinese populations [38-40]. But few studies have been conducted to review those HRQoL measures that were developed for Chinese populations.

Consequently, a review was conducted to identify Chinese-developed HRQoL measures and to synthesise their contents. There were two main objectives of the review: 1) to establish a conceptual framework summarising concepts that comprise HRQoL assessment in China; 2) to compare similarities and differences in HRQoL concepts between Chinese-developed HRQoL measures and Western-developed HRQoL measures. This would enable exploration of cultural differences in defining and measuring health between the East and the West.

\section{Methods:}

\section{Identifying Chinese-developed HRQoL measures}

Studies included if they reported (1) HRQoL measures that were developed in a Chinese cultural setting with the aim of assessing health for Chinese people and (2) to be generic. Studies excluded if they (1) were originally developed in other countries 
and translated into Chinese, (2) lacked validity evidence, or (3) were developed for diagnosis rather than health assessment.

Two Chinese databases (CNKI and WANFANG), as well as four English databases (PsycINFO, Global Health, Ovid MEDLINE and EMBASE) were searched for published papers. Examples of searching strategies (in the English language) in Medline and (in the Chinese language) in CNKI are presented in Appendix. Reference lists of all identified reports and articles were searched for additional studies. Further, hand searching was conducted in six Chinese key medical journals and Google Scholar was used to search for additional papers.

After searching in the databases, citations along with the abstracts were imported in EndNote reference management software, where duplications were deleted. Two researchers independently screened citations based on the inclusion and exclusion criteria. Differences in the selection process were resolved through discussions or by consulting the third reviewer. Information about those studies that met the criteria was extracted by one reviewer and was checked by the second reviewer. In the case of unclear or missing data, publication authors were contacted.

Selecting Non-Chinese-developed measures included for comparison Chinese versions of EQ5D, SF-36 and WHOQOL-100 were included as comparators to the identified Chinese-developed measures. EQ-5D and SF-36 were two HRQoL measures that were originally developed in Western countries [13,41]. WHOQOL was developed cross-culturally in various field centres around the world and claimed 
to be eligible to be used in different cultural settings [5]. They are widely applied in China and are often regarded as a "golden standard" in validation studies of Chinese new measures [42], therefore, they were chosen for comparison with Chinesedeveloped HRQoL measures.

\section{Content analysis and framework analysis}

Content analysis was used to attain a condensed and broad description of the identified HRQoL measures. Both inductive and deductive approaches were applied. Deductively, the terminology used in the Patient-Reported Outcomes Measurement Information Systems (PROMIS) framework was utilised in labelling each question in the measures. Inductively, when items could not be assigned in the pre-defined framework, new concepts or categories were generated.

Reviewers obtained a copy of the identified HRQoL measures and entered each question item into an Excel spreadsheet. Three members of the research team worked independently and performed the initial coding. Researchers considered each item in turn and coded it with a concept, then classified it into a sub-domain, then a domain. For example, one item in the Current Perceived Health Questionnaire-42 states "It is hard to keep your mind on things”. This item was labelled as “concentration” and classified in the sub-domain "cognitive function" as well as the domain "mental health”. If one item represented more than one concept or domain, then the item was split and coded as two conceptual items; such as "anxiety and depression" from EQ5D, it was coded as two concepts. 
Once all items had been coded, the three researchers compared results to reach a consensus on any discrepancies. A conceptual model, containing domains, subdomains and concepts, was then constructed. Content of the Chinese and Western HRQoL measures was then compared.

\section{Results}

A total of 4535 articles were identified by our search strategy (see Fig. 1) with 34 articles satisfying the inclusion criteria. Eight measures were found with satisfactory psychometric properties [37, 43-46] and with full questionnaires available for this review. Table 1 shows the characteristics of the 8 Chinese-developed HRQoL measures. Four of them were developed from a TCM perspective, while the remaining four measures were not. 
Table 1. Characteristics for the included Chinese and Western-developed measures

\begin{tabular}{|c|c|c|c|}
\hline $\begin{array}{l}\text { Name, Author(s), } \\
\text { Publication year }\end{array}$ & Research Aims & Development Methods & $\begin{array}{l}\text { No. of } \\
\text { items }\end{array}$ \\
\hline \multicolumn{4}{|c|}{ Chinese-developed measures $(n=8)$} \\
\hline $\begin{array}{l}\text { Quality of Life Inventory Li } \\
\text { et al, } 1995 \text { [43] }\end{array}$ & $\begin{array}{c}\text { To evaluate quality of life among general } \\
\text { populations in communities in China }\end{array}$ & $\begin{array}{l}\text { literature review, expert opinions } \\
\text { and interview residents }\end{array}$ & 99 \\
\hline $\begin{array}{c}\text { Current Perceived Health } \\
\text { Questionnaire-42, R. } \\
\text { Fielding and J. Li, 1997 [44] }\end{array}$ & To measure current perceived health & $\begin{array}{l}\text { literature review and interview } \\
\text { with outpatients and inpatients }\end{array}$ & 42 \\
\hline $\begin{array}{c}\text { Self-rated Health } \\
\text { Measurement Scale, Xu et } \\
\text { al, } 2000 \text { [45] }\end{array}$ & $\begin{array}{l}\text { To develop self-rated health questionnaires } \\
\text { that are suitable for the Chinese situation }\end{array}$ & $\begin{array}{l}\text { Literature review and expert } \\
\text { opinions }\end{array}$ & 48 \\
\hline QoL-35, Wu et al, 2005 [46] & $\begin{array}{l}\text { To develop a QoL scale that is suitable for } \\
\text { the Chinese general population }\end{array}$ & $\begin{array}{l}\text { Literature review and expert } \\
\text { opinions }\end{array}$ & 35 \\
\hline $\begin{array}{l}\text { Chinese QoL Instrument, } \\
\text { Liu et al, } 2007 \text { [35] }\end{array}$ & $\begin{array}{l}\text { To develop a QoL instrument reflecting } \\
\text { Chinese culture and TCM knowledge }\end{array}$ & $\begin{array}{l}\text { Literature review and expert } \\
\text { opinions }\end{array}$ & 50 \\
\hline $\begin{array}{l}\text { Health Scale of TCM, Wu } \\
\text { and Lai, } 2007 \text { [47] }\end{array}$ & $\begin{array}{c}\text { To develop a health scale based upon TCM } \\
\text { theory }\end{array}$ & $\begin{array}{l}\text { Interview with professionals and } \\
\text { non-professionals }\end{array}$ & 88 \\
\hline ChPRO, Li et al, 2007 [48] & $\begin{array}{l}\text { To develop a PRO measure that can be used } \\
\text { for the curative effect appraise of the TCM }\end{array}$ & $\begin{array}{c}\text { Expert group and interview with } \\
\text { patients }\end{array}$ & 61 \\
\hline $\begin{array}{l}\text { TCM-HSS, Liu et al, } 2008 \\
\text { [49] }\end{array}$ & $\begin{array}{c}\text { To develop a health status scale of TCM } \\
\text { based on Chinese culture and TCM }\end{array}$ & $\begin{array}{l}\text { Literature review and expert } \\
\text { opinions }\end{array}$ & 30 \\
\hline \multicolumn{4}{|c|}{ Non-Chinese-developed measures $(n=8)$} \\
\hline EQ-5D [50] & $\begin{array}{l}\text { To provide a simple, generic measure of } \\
\text { health for clinical and economic appraisal }\end{array}$ & Review and expert opinions & 5 \\
\hline WHOQOL100 [5] & $\begin{array}{l}\text { To assesses individuals' perceptions of their } \\
\text { position in life in the context of the culture } \\
\text { and value systems in which they live and in } \\
\text { relation to their goals, expectations, } \\
\text { standards and concerns }\end{array}$ & $\begin{array}{c}\text { Expert opinions, Focus groups, } \\
\text { expert and lay question writing } \\
\text { panel }\end{array}$ & 100 \\
\hline SF-36 [13] & $\begin{array}{c}\text { To develop a patient self-reporting generic } \\
\text { HRQoL measure }\end{array}$ & Expert opinions & 36 \\
\hline
\end{tabular}


Table 2. Number of items classified in each sub-domain

\begin{tabular}{|c|c|c|c|c|c|c|}
\hline \multirow{2}{*}{$\begin{array}{c}\text { Major domain/ } \\
\text { sub-domain }\end{array}$} & \multicolumn{3}{|c|}{ Number of items ${ }^{a}$} & \multirow{2}{*}{$\begin{array}{c}\text { Number of } \\
\text { items in } \\
\text { Western } \\
\text { measures }\end{array}$} & \multirow{2}{*}{$\begin{array}{c}\text { Total } \\
\text { number of } \\
\text { items }^{\mathrm{b}} \\
\mathrm{N}(\%)\end{array}$} & \multirow{2}{*}{$\begin{array}{c}\text { Number of } \\
\text { questionnaire } \\
\text { items }^{\mathrm{c}}\end{array}$} \\
\hline & $\begin{array}{l}\text { Items in } \\
\text { MM }\end{array}$ & $\begin{array}{l}\text { Items in } \\
\text { TCM }\end{array}$ & Sub-total & & & \\
\hline \multicolumn{7}{|l|}{ Physical health $(n=250)$} \\
\hline Physical symptoms & 31 & 70 & 101 & 19 & $120(22.43)$ & 116 \\
\hline Physical function & 47 & 16 & 63 & 30 & 93(17.38) & 91 \\
\hline Appearance & 1 & 16 & 17 & 4 & $21(3.93)$ & 21 \\
\hline Physical adaption & 0 & 15 & 15 & 1 & 16(2.99) & 14 \\
\hline \multicolumn{7}{|l|}{ Mental health $(n=161)$} \\
\hline Negative affect & 22 & 31 & 53 & 9 & 62(11.59) & 61 \\
\hline Positive affect & 16 & 13 & 29 & 17 & $46(8.60)$ & 46 \\
\hline Cognitive function & 13 & 19 & 33 & 3 & $36(6.73)$ & 33 \\
\hline Mental adaption & 1 & 16 & 17 & 0 & $17(3.18)$ & 17 \\
\hline \multicolumn{7}{|l|}{ Social health $(n=90)$} \\
\hline Social relation & 44 & 2 & 46 & 9 & $55(10.28)$ & 55 \\
\hline Social function & 14 & 2 & 16 & 16 & $32(5.98)$ & 32 \\
\hline Social adaption & 3 & 0 & 3 & 0 & $3(0.56)$ & 3 \\
\hline \multicolumn{7}{|c|}{ General health assessment $(n=34)$} \\
\hline Global health & 13 & 4 & 17 & 9 & $26(4.86)$ & 26 \\
\hline $\begin{array}{l}\text { General life } \\
\text { satisfaction }\end{array}$ & 3 & 2 & 5 & 3 & $8(1.50)$ & 8 \\
\hline Total number (\%) & 208(39.79) & 206(35.51) & $415(75.30)$ & $120(24.70)$ & $535(100)$ & 523 \\
\hline
\end{tabular}

a Items have been divided by Chinese questionnaires developed based on Traditional Chinese Medicine (TCM) and Non-TCM Medicine (Modern Medicine MM) theories

b Total number of health items, each of which can represent one separate aspect of health.

${ }^{\mathrm{c}}$ Total number of single questions that were included in the identified questionnaires. 
Table 3. Number of questionnaires covered each concept

Appearance

Physical adaption

Negative affect

Positive affect

Cognitive function

Mental adaption

$\begin{array}{cccccc}\text { Sleep } & 4 & 4 & 8 & 1 & 9 \\ \text { Usual activities } & 3 & 0 & 3 & 3 & 6 \\ \text { Verbal expression } & 0 & 2 & 2 & 0 & 2 \\ \text { Vision } & 2 & 0 & 2 & 0 & 2 \\ \text { Complexion } & 0 & 2 & 2 & 0 & 2 \\ \text { Body image } & 1 & 1 & 2 & 1 & 3 \\ \text { Body weight } & 0 & 1 & 1 & 0 & 1 \\ \text { Eye spirit } & 0 & 3 & 3 & 0 & 3 \\ \text { Lip } & 0 & 2 & 2 & 0 & 2 \\ \text { Spirit } & 0 & 1 & 1 & 0 & 3 \\ \text { Weather adaption } & 0 & 3 & 3 & 0 & 3 \\ \text { Susceptibility } & 0 & 2 & 2 & 1 & \end{array}$

Mental health

\begin{tabular}{|c|c|c|c|c|}
\hline Anger & 0 & 3 & 3 & \\
\hline Anxiety & 3 & 3 & 6 & \\
\hline Depression & 3 & 3 & 6 & \\
\hline Fear & 1 & 2 & 3 & \\
\hline Loneliness & 3 & 1 & 4 & \\
\hline General negative affect & 3 & 0 & 3 & \\
\hline Nervous & 3 & 1 & 4 & \\
\hline Worry & 2 & 2 & 4 & \\
\hline Calm & 1 & 2 & 3 & \\
\hline Meaning and purpose & 2 & 1 & 3 & \\
\hline General positive affect & 4 & 3 & 7 & \\
\hline Sense of security & 2 & 2 & 4 & \\
\hline Self-efficacy & 4 & 1 & 5 & \\
\hline Concentration & 4 & 3 & 7 & \\
\hline Decision making & 1 & 0 & 1 & \\
\hline Memory & 3 & 3 & 6 & \\
\hline Reaction & 1 & 3 & 4 & \\
\hline Thinking & 2 & 3 & 5 & \\
\hline Emotion control & 0 & 2 & 2 & \\
\hline Mental response & 1 & 2 & 3 & \\
\hline
\end{tabular}




\begin{tabular}{ccccccc}
\multicolumn{2}{c}{ Social health } & & & & \\
Social relation & Social relation & 4 & 1 & 5 & 2 & 7 \\
& $\begin{array}{c}\text { Social support } \\
\text { Social function }\end{array}$ & 4 & 1 & 5 & 1 & 6 \\
& $\begin{array}{c}\text { Ability to perform social } \\
\text { activities }\end{array}$ & 1 & 0 & 1 & 1 & 2 \\
& $\begin{array}{c}\text { Ability to perform social } \\
\text { roles }\end{array}$ & 4 & 2 & 6 & 2 & 8 \\
Social adaption & $\begin{array}{c}\text { Recreation \& leisure } \\
\text { Social adaption }\end{array}$ & 1 & 0 & 1 & 1 & 2 \\
General life & General health assessment & & & 2 \\
satisfaction & General life satisfaction & 3 & 2 & 5 & 1 & 6 \\
Global health & Global health & 3 & 3 & 6 & 3 & 9 \\
\hline
\end{tabular}

A total number of 571 questionnaire items were documented and 48 items were excluded because they were classified as respondents' external environment conditions (health determinants) rather than components of health status, while this paper is only interested in exploring the content that was embraced in individuals’ subjective assessment of health. This resulted in 523 questionnaire items that were recorded. Since there were ten questionnaire items with two concepts and one item containing three concepts, therefore a total number of 535 conceptual items were coded to define the concepts within the framework. Each concept was assigned to a sub-domain and a domain. The number of questionnaire items in each sub-domain is presented in Table 2.

Figure 2 shows a model of HRQoL developed from the content analysis of the 11 included HRQoL measures. The conceptual framework consisted of 4 domains, 14 
sub-domains and 64 concepts. Content of the conceptual framework is summarised in Table 2 and is explained in detail in the following sub-sections.

\section{Physical health}

Physical health was the most frequently assessed domain, with 250 items (47\%) coded as concepts belonging to this domain. There were four sub-domains for physical health - physical symptoms, physical function, appearance and physical adaption - containing 35 concepts in total. Physical symptoms (22\%) and physical function (17\%) were two most commonly mentioned sub-domains and they were addressed in all the eleven measures.

Various physical symptoms were identified from the measures. Some of them were covered by both Western and Chinese measures, while many concepts were exclusively mentioned in Chinese-developed HRQoL measures. Appetite, for example, was assessed by all the eight Chinese measures, while none of the Western counterparts included such item. Similarly, several specific physical signs, such as urination, stool, mouth feeling, chest feeling and sweat, were included in Chinese questionnaires, especially those integrating TCM knowledge, but they were not frequently considered from a Western perspective.

Both Western measures and Chinese-developed measures covered concepts regarding physical function. Some of the concepts were simultaneously identified in the two groups of measures, including mobility, physical activities (specifically referring to those activities that required considerable physical effort, such as lifting heavy 
things), self-care, sexual function and satisfaction, sleep and usual activities. It may be worth noting that sleep was found in all the Chinese-developed health measures, while only WHOQOL100 included this concept. Another difference in content between the Western and Chinese measures was that hearing, verbal expression and vision were only mentioned in Chinese measures, while learning was exclusively asked in WHOQOL100.

Appearance and physical adaption were two sub-domains that were mainly covered by “Chinese-specific” concepts. Appearance was understood as a person’s external appearance. This sub-domain consisted of concepts that are visible and can be observed by other people: things like facial complexion, eye spirit, body weight, lip and spiritual appearance were included in several Chinese-developed questionnaires for health appraisal. Some of these concepts were Chinese idioms and purely came from Traditional Chinese knowledge, such as “eye spirit” and "spiritual appearance”. "Spirit" is a central notion in traditional Chinese knowledge, yet it seems to be elusive, with various meanings and is hard to be defined even in Chinese [51,52]. In a broad sense, it is understood as the external manifestation of one’s life activities[51]. In a narrower sense, “spirit” is mainly referred to people’s consciousness, mind, thoughts and/or vitality, emphasising on different meanings in different contexts [5355]. "Having eye spirit” may be understood as one’s eyes could focus on things and are with a bright vision; "spiritual appearance" is the overall appearance of an individual. As it was illustrated by Deng and Guo [56], when one had spirit, it means “one’s mind is clear, vision is bright, talking is clear, complexion is glowing, facial 
expression is natural, response is quick, movement is agile, breathing is smooth and steady...” This quote suggests that spirit is closely associated with physical symptoms (including complexion and eyesight), physical function (verbal expression) as well as cogntive function (reaction and concentration), which were often included as questionnaire items to assess health in thoese TCM measures.

Several Chinese measures included items on relationships between an individual and his/her surrounding environment, aiming to assess a person's physical health status under certain circumstances. Such questions were classified as concepts in "physical adaption”. For example, some Chinese questionnaires asked whether weather/season changes affected one’s body (“weather adaption”). There were other questions asking whether one is afraid of cold/hot or whether someone is susceptible to diseases when the weather changes. They considered an individual's susceptibility to his/her external surroundings and were grouped as "susceptibility”. Among the three Western questionnaires, only SF-36 contained a question asking if a person seemed to get sick easier than others. Although the question was coded as "susceptibility", it did not address influences caused by the environment.

\section{Mental health}

A total of 158 items (30\%) were classified in the mental health domain, which was consisted of negative affect, positive affect, cognitive function and mental adaption. The first three sub-domains were included by more than $80 \%$ of the questionnaires. 
Most frequently mentioned concepts included depression, mental response, anxiety, general positive affect and concentration.

Measures developed from both Chinese and Western perspectives covered negative mental symptoms including anxiety, depression, loneliness, nervous and worry, while anger and fear were two concepts only found in Chinese ones. As for positive mental symptoms, items such as mental state of calmness, meaning and purpose, sense of security and self-efficacy were referred to in both Chinese and Western measures.

The cognitive function can be understood as the abilities to perform mental activities and was, therefore, classified as a subdomain in mental health. Concentration, decision-making ability and memory were included in both Chinese and Western contexts. Among them, concentration was paid particular attention by the Chinese measures, when seven out of eight Chinese questionnaires included this concept. Several Chinese-developed measures asked questions regarding reaction and thinking ability, while none of the three Western counterparts covered such concepts in assessing health.

Similar to the concept "physical adaption”, "mental adaption” was identified as an exclusive Chinese aspect in the mental health domain. "Mental adaption” was about the potential influences of external surrounding on one's mental states. Under this sub-domain, two concepts, “mental response” and “emotion control” were recognised. More specifically, “mental response” assessed how a person’s reacted, mentally, under a specified circumstance. For example, HSTCM included a question "Will you 
be nervous when you are with unfamiliar people or in an unfamiliar environment?” This question illustrated a circumstance and assumes that such an environment would affect a person's mental state. By asking whether a person feel nervous or not under an unfamiliar condition, respondents' ability to mentally adapt to this environment was assessed. "Emotion control” was another concept in this sub-domain and was also only mentioned in Chinese-developed measures. Two Chinese measures asked "can you control your emotions?” to assess the ability to adjust emotions to external influences.

\section{Social health}

The domain of social health included three sub-domains: social relation, social function and social adaption, with 90 items (17\%) in total. Social relation and social function were assessed by around half of the questionnaires. Questions about social relations and social supports appeared 30 times and 25 times respectively. Ability to perform social roles, in the social function domain, was another frequently mentioned concept $(n=23)$.

Both Western and Chinese HRQoL measures addressed the social domain of health and their included concepts were similar to each other.

The sub-domain of social relation consisted of questions concerning the quality of social relationships and the condition of social support. They were both covered in the majority of Chinese questionnaires and were also mentioned by Western questionnaires. 
In the aspect of social function, six out of the eight Chinese HRQoL measures assessed individuals' ability to perform social roles, such as the ability to work and the ability to maintain family responsibilities. Several Chinese measures also included questions on the ability to perform social activities or the state of recreation and leisure activities. Those concepts were identical to those in Western measures, such as SF-36 and WHOQOL100.

"Social adaption" was identified as a sub-domain and was only included in Chinese measures. Similar to physical adaption and mental adaption, it considered one’s relationships with the external environment and assessed a person's ability to adapt to such an environment, but from a social perspective. For example, HSTCM asked whether a person is able to fit into a new working or studying environment within a limited time. None of the three Western measures included similar questions.

\section{General health assessment}

The general health assessment domain (34 items, 6\%) included items pertaining to assess individuals' overall health status. There were mainly two types of questions in this domain, one asked about general life satisfaction, the other assessed health status. The majority of the identified questionnaires, both Chinese and Western, contained questions that assess the overall condition. 


\section{Discussion}

A conceptual model combining health items from both Chinese-developed and Western-develop HRQoL measures was developed. Both Western-developed and Chinese-developed measures involved similar organising frameworks: physical, mental, social and general health domains and consisted of similar sub-domains and health concepts for measuring health. More than half of the identified concepts (38/68) were included in both Western and Chinese measures.

Despite these similarities, there were some clear differences. The first and foremost difference was that TCM knowledge seemed to play an important role in HRQoL assessment in China. Among the eight Chinese-developed HRQoL measures that were identified, half of them were developed combining TCM knowledge. This may be because TCM, working alongside Western (Modern) Medicine, has played an active role in the Chinese health care system $[57,58]$.

TCM has its unique theoretical and practical perception of health, therefore, questionnaires developed following TCM knowledge system had distinctive characteristics compared to non-TCM developed Chinese measures and those Western HRQoL measures. TCM questionnaires had items mainly on symptom status to assess the health status of people. Questions about physical and mental symptoms constituted a great portion of the TCM questionnaires, while there were fewer questions about physical and social functional status. It may be because the TCM theory encompasses a holistic concept of health, which means body components are 
supposed to be connected and interact with each other, thus it was believed that a small sign of the body is able to reflect an individual's health status [55]. In practice, typical diagnostic methods of TCM, involving inspection, listening, smelling, as well as pulse taking, are also about examining trivial symptoms of an individual. For example, TCM practitioners tend to observe patients' face colour and make inquiries about patients' stool, urine, mouth feelings, sweat and skin. Some of these specific items were recognised in the TCM health questionnaires. It seems that they are common factors to detect health status from a TCM perspective, therefore these symptom-relevant questions were frequently mentioned by those TCM health measures.

Concepts like "Weather adaption”, “Emotion control” and "Social adaption” were also mainly referred to in TCM questionnaires and were not part of the descriptive systems of the Western HRQoL measures. These concepts addressed the ability to adapt as a component in measuring health. It may be inspired by those Chinese old sayings "Harmony between man and nature", which also indicates a holistic view of health. This phrase suggests an idea that one can reach a dynamic harmonious state by being adaptive to changes of natural or social environments [59], therefore a person's adaptability is associated with his/her health state. For example, a person who has a better immune system and is more likely to adapt to weather changes is supposed to be in a better physical health state. While it is also believed that being able to adapt to the external social environment better, such as the working environment, one could expect a better social wellbeing state. The idea of defining health as the ability to 
adapt has been proposed by some Western scholars [60-62] but seems to be rarely used in Western HRQoL measures.

Aside from those concepts that may be considered TCM features, there were health dimensions that were emphasised by Chinese health measures but were less apparent in the Western HRQoL measures. Sleep was agreed to be an applicable health indicator among the eight Chinese-developed measures. The connection between health and sleep was investigated and confirmed in various Chinese empirical studies [63-65]. Many Chinese traditional Chinese idioms show that sleep is an important factor in staying in health, for example, the saying "One good sleep is better than

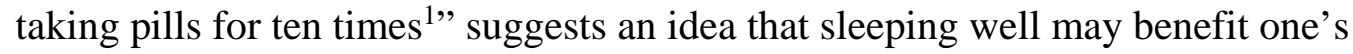
health condition. The importance of sleep is also highlighted in TCM theory, where it is believed that sleep can help to protect a person’s “Qi” and restore one’s energy [66,67]. Appetite was another concept introduced in all the identified Chinese health measures, but none of the western counterparts included such item. The Chinese dining culture may have originated from the fact that China is a traditional agricultural country with a large population, where food production and consumption is considered as a fundamental and principal theme by Chinese governors as well as ordinary Chinese people $[68,69]$. It can then illustrate how individuals' desire to have food was considered to be one of the indicators in examining health, given the important role “Appetite” plays in a Chinese cultural setting.

“吃药十付, 不如独宿一夜” 
Therefore, health concepts, such as “spirit”, “complexion”, “weather adaptation” and “social adaption” were exclusively introduced in Chinese literature, but which were not covered in Western measures. Additionally, items like appetite and sleep were frequently referred to in Chinese-developed questionnaires and by Chinese interviewees but were less likely to be emphasised in those Western measures such as EQ-5D and SF-36. These "Chinese-characteristic" health concepts demonstrated potential cultural differences in defining health between the East and the West. Applying translated Western HRQoL measures may facilitate researchers in nonEnglish speaking countries to conduct HRQoL studies in some respects, but as health is a culturally relevant concept, factors that are relevant and important in defining and measuring health in a Western setting, may not necessarily be relevant or important in China [35]. Therefore, it is then worth considering such cultural differences when selecting HRQoL measures for trials or clinical practices as well as health research involving participants with different cultural background. As shown in the current study, the East and the West may share a similar structure and most concepts in measuring health, but unique "Chinese specific” concepts exist. Similar organising frameworks and key health items from a Western measure may be kept, while concepts reflecting Chinese culture may also be important to be considered in assessing health among Chinese populations. Our result implies the necessity of further examining the legitimacy of applying the Western-developed HRQoL measures in a Chinese cultural setting. 
On the other hand, despite HRQoL measures have been developed and validated for Chinese populations, most of these measures seem to be with much less significance or application, compared to those Western measures such as EQ-5D and SF-36 in Chinese HRQoL research. This may be because Chinese researchers are more likely to trust those Western HRQoL measures which are supported by accumulating evidence of validity and have already been used widely. In addition, although Chinese-developed HRQoL measures seem to be more sensitive to the Chinese culture and may outperform those Western-developed HRQoL measures in assessing health China, being Chinese culture specific can limit their application, especially in cross-country comparative studies or international clinical trials. Meanwhile, many Chinese-developed HRQoL measures, especially those developed based on TCM theories, have not provided a well-established and widely-recognised construct of health and may be applicable to TCM practice or research only. Additionally, many Chinese-developed HRQoL measures (mainly TCM measures) are often regarded as just a diagnostic tool in clinical practice, while they have been rarely used to monitor and track changes of the clinical outcomes. These challenges and misunderstandings of Chinese-developed HRQoL measures have become obstacles to their widely use. With much efforts have been devoted in developing HRQoL measures for Chinese populations, the current study implies the need to further refine and develop an agreeable conceptual framework of health for use among Chinese populations, before considering inventing new HRQoL measures with limited potential for widely use. We would also recommend further research to guide and promote the use of Chinese- 
developed HRQoL measures. Because for those Chinese-developed HRQoL measures with sufficient content validity and quality, they may be able to collect better quality health status information of Chinese populations and are supposed to lead to better clinical decisions, policymaking and resource allocation.

\section{Limitation}

The review only focused on those papers that had reported development and/or validation processes. It is possible that some Chinese generic HRQoL measures were excluded due to omission of descriptions of measure development. Therefore, there was a risk that such eligible questionnaires may be neglected. However, it is not practical to include all Chinese studies involving HRQoL measures, since a significantly large number of papers had implemented Western HRQoL measures to assess health among Chinese populations.

Meanwhile, this study only selected three non-Chinese HRQoL measures for comparison. Various Western-developed HRQoL measures are available but it would be not practical to include all those health questionnaires in this study. EQ-5D, SF-36 and WHOQOL100 were selected as examples for comparison because they have been most frequently used in China for assessing HRQoL.

Another limitation related to coding biases since the process involved researchers' own subjective understandings. The PROMIS standards were applied in the coding process and concepts were blindly coded by three researchers to try to control such biases. 
Further, the study conducted a secondary data analysis to explore how health is defined and measured in China. It may not include all health concepts that are considered important by Chinese people. Further empirical studies are needed to better understand how health is described among Chinese populations.

\section{Conclusion}

This paper describes health concepts that form part of questionnaires used to measure HRQoL in a Chinese cultural setting and proposes a conceptual framework of health based on these synthesised materials. It presents an overview of how health can be defined and measured in HRQoL researches in Eastern culture and demonstrates that Chinese-developed health measures include specific features relating to the Chinese culture and that there are cultural differences in Eastern and Western approaches to defining and measuring health. Western questionnaires considered for use among Chinese populations will need careful scrutiny and potential modification to improve their capacity to measure health status when they are applied in a Chinese cultural setting.

\section{References:}

1. Guyatt G, Feeny D, Patrick D. Issues in quality-of-life measurement in clinical trials. Controlled Clinical Trials. 1991;12(4):S81-S90.

2. Kaplan RM, Bush JW. Health-related quality of life measurement for evaluation research and policy analysis. Health psychology. 1982;1(1):61.

3. US Department of Health and Human Services Food and Drug Administration. Patient-Reported Outcome Measures: Use in Medical Product Development to Support Labeling Claims: Guidance for Industry. https://www.fda.gov/regulatory-information/search-fda-guidance- 
documents/patient-reported-outcome-measures-use-medical-productdevelopment-support-labeling-claims. 2009. [Accessed July 15th, 2020].

4. Shumaker SA, Naughton MJ. The international assessment of healthrelated quality of life: a theoretical perspective. In: Shumaker SA, Berzon $\mathrm{RA}$, eds. The international assessment of health-related quality of life: theory, translation, measurement and analysis. Oxford: Rapid Communications; 1995:3-10.

5. The WHOQOL Group. The World Health Organization quality of life assessment (WHOQOL): Position paper from the World Health Organization. Social Science \& Medicine. 1995;41(10):1403-1409.

6. Bowling A. Health-related quality of life: conceptual meaning, use and measurement. In: Measuring disease: a review of disease-specific quality of life measurement scales. 2nd ed. Buckingham: Open University Press; 2001:1-22.

7. Fayers PM, Machin D. Quality of life: the assessment, analysis and interpretation of patient-reported outcomes. 2nd ed. England: John Wiley \& Sons; 2013.

8. Schipper H, Clinch JJ, Olweny CL. Quality of life studies: definitions and conceptual issues. In: Spilker B, ed. Quality of life and pharmacoeconomics in clinical trials. 2nd ed. New York: Lippincott-Raven Press; 1996.

9. Ware JE. Standards for validating health measures: definition and content. Journal of Chronic Diseases. 1987;40(6):473-480.

10. Wilson IB, Cleary PD. Linking clinical variables with health-related quality of life: a conceptual model of patient outcomes. Jama. 1995;273(1):59-65.

11. Karimi M, Brazier J. Health, health-related quality of life, and quality of life: what is the difference? Pharmacoeconomics. 2016;34(7):645-649.

12. Bowling A. Measuring health: A review of quality of life measurement scales. 3rd ed. Maidenhead: Open University; 2004.

13. Ware J, Sherbourne CD. The MOS 36-Item Short-Form Health Survey (SF36): I. Conceptual Framework and Item Selection. Medical Care 1992.

14. Cunillera O, Tresserras R, Rajmil L, et al. Discriminative capacity of the EQ-5D, SF-6D, and SF-12 as measures of health status in population health survey. Quality of Life Research. 2010;19(6):853-864.

15. Brazier J, Roberts J, Deverill M. The estimation of a preference-based measure of health from the SF-36. Journal of health economics. 2002;21(2):271-292.

16. Makai P, Brouwer WB, Koopmanschap MA, Stolk EA, Nieboer AP. Quality of life instruments for economic evaluations in health and social care for older people: a systematic review. Social science \& medicine. 2014;102:83-93.

17. Hill MR, Noonan VK, Sakakibara BM, Miller WC. Quality of life instruments and definitions in individuals with spinal cord injury: a systematic review. Spinal cord. 2010;48(6):438. 
18. Kalantar-Zadeh K, Kopple JD, Block G, Humphreys MH. Association among SF36 quality of life measures and nutrition, hospitalization, and mortality in hemodialysis. Journal of the American Society of Nephrology. 2001;12(12):2797-2806.

19. Guillemin F, Bombardier C, Beaton D. Cross-cultural adaptation of healthrelated quality of life measures: literature review and proposed guidelines. Journal of Clinical Epidemiology. 1993;46(12):1417-1432.

20. Bowden A, Fox-Rushby JA. A systematic and critical review of the process of translation and adaptation of generic health-related quality of life measures in Africa, Asia, Eastern Europe, the Middle East, South America. Social Science \& Medicine. 2003;57(7):1289-1306.

21. Acquadro C, Conway K, Hareendran A, Aaronson N. Literature review of methods to translate health-related quality of life questionnaires for use in multinational clinical trials. Value in Health. 2008;11(3):509-521.

22. Herdman M, Fox-Rushby J, Badia X. 'Equivalence' and the translation and adaptation of health-related quality of life questionnaires. Quality of Life Research. 1997;6(3):237-247.

23. Herdman M, Fox-Rushby J, Badia X. A model of equivalence in the cultural adaptation of HRQoL instruments: the universalist approach. Quality of Life Research. 1998;7(4):323-335.

24. Stewart AL, Napoles-Springer A. Health-related quality-of-life assessments in diverse population groups in the United States. Medical Care. 2000:II102-II124.

25. Levesque A, Li HZ, Bohémier M. Cultural variations in health conceptions: A qualitative approach. Pimatisiwin. 2013;11(2):215-229.

26. Bowling A. Social research on health: sociological and psychological concepts and approaches. In: Research methods in health: investigating health and health services. 4th ed. Maidenhead: Open University Press; 2014:17-43.

27. Parker M, Fox-Rushby J. International comparisons in health related quality of life: acquiescence in academia. In: Shumaker SA, Richard B, eds. The international assessment of health-related quality of life: theory, measurement \& analysis. . Oxford: Rapid Communications; 1995.

28. Prior L, Chun PL, Huat SB. Beliefs and accounts of illness. Views from two Cantonese-speaking communities in England. Sociology of Health \& Illness. 2000;22(6):815-839.

29. Liu JE, Mok E, Wong T. Perceptions of Chinese cancer patients of the favorable and unfavorable words conveyed by their social support providers. Cancer Nursing. 2005;28(5):348-354.

30. Chen AW, Kazanjian A, Wong H. Why do Chinese Canadians not consult mental health services: Health status, language or culture? Transcultural Psychiatry. 2009;46(4):623-641. 
31. Xiang YT, Chiu HF, Ungvari GS. Quality of life and mental health in Chinese culture. Current Opinion in Psychiatry. 2010;23(1):43-47.

32. Wong W, Lam CLK, Wong VT, Yang ZM, Ziea ETC, Kwan AKL. Validation of the constitution in chinese medicine questionnaire: does the traditional chinese medicine concept of body constitution exist? Evidence-based Complementary and Alternative Medicine. 2013:481491.

33. Leung B, Luo N, So L, Quan H. Comparing three measures of health status (perceived health with Likert-type scale, EQ-5D, and number of chronic conditions) in Chinese and white Canadians. Medical Care. 2007:610-617.

34. Cnossen MC, Polinder S, Vos PE, et al. Comparing health-related quality of life of Dutch and Chinese patients with traumatic brain injury: Do cultural differences play a role? Health and Quality of Life Outcomes. 2017;15 (1):72.

35. Liu FB, Zhao L, Lang JY, Lin LZ, Liang GH, Fang JQ. Development of the Chinese Quality of Life Instrument. Journal of Clinical Rehabilitative Tissue Engineering Research. 2007;11(52):10492-10495.

36. Liu F-b, Hou Z-k, Yang Y-y, et al. Literature review and analysis of the development of health outcomes assessment instruments in Chinese medicine. Journal of Integrative Medicine. 2013;11(2):80-89.

37. Yu C, Sun Y, He L, Liu B. Traditional Chinese Medicine Instruments for quality of life: a systematic review of psychometric evidence. Chinese Journal of Traditional Chinese Medicine and Pharmacy. 2015;30(5):16311635.

38. Wang F, Li H, Ma A. Study on the application of General Utility Scale on Chinese populations. Chinese Journal of Drug Evaluation. 2015;32(4):244247.

39. Zhou T, Guan H, Liu G, Ma A. Health-related quality of life for the general population in China: a systematic review. Chinese Health Service Management. 2016;33(8):621-623.

40. Zhou T, Guan H, Liu G, Ma A. Health-related quality of life for disease population in China based on EQ-5D: a systematic review. Chinese Journal of Evidence-Based Medicine. 2016;16(2):135-142.

41. Prieto L, Novick D, Sacristan JA, Edgell ET, Alonso J, Group SS. A Rasch model analysis to test the cross-cultural validity of the EuroQoL-5D in the Schizophrenia Outpatient Health Outcomes Study. Acta Psychiatrica Scandinavica, Supplementum. (416):24-29.

42. Yu C, Sun Y, He L, Bai W, Liu B. Comparative study on the concepts of health related quality of life from TCM and modernized medicine measures. China Journal of Traditional Chinese Medicine and Pharmacy. 2016;31(11):4773-4778.

43. Li L, Young D, Wei H, et al. Quality of Life Research in a Community Population - the Development of QOLI. Chinese Mental Health Journal. 1995;9(5):227-231. 
44. Fielding R, Li J. A validation of the concept of current perceived health and the Current Perceived Health-42 (CPH-42) questionnaire. Quality of Life Research. 1997;6(1).

45. Xu J, Wang B, Hu M, Yang B, Chen H, Xie Y. The development and evaluation of self-rated health measurement scale-prior test version. Chinese journal of behavioral medical science. 2000;9(01):65-68.

46. Wu Y, Xie G, Li Y, et al. The development and Assessment on the general quality of life intrument for Chinese people. Chinese Journal of Epidemiolodgy. 2005;26(10):751-756.

47. Wu D, Lai S, Guo X, Wen Z, Liang W, Yang X. Establishment and initial evaluation of Health Scale of Traditional Chinese Medicine. Chinese Journal of Integrative Medicine. 2007;27(9):847-850.

48. Li X. The development and evaluation of Chinese PRO Scale. Guangzhou, Guangzhou University of Chinese Medicine; 2007.

49. Liu F-B, Lang JY, Zhao L, Fang J, Leung K-F, Lin L. Development of Health Status Scale of Traditional Chinese Medicine(TCM-HSS). Journal of Sun Yat-Sen University (Medical Sciences). 2008;29(3):332-336.

50. The EuroQol Group. EQ-5D-5L user guide: https://euroqol.org/docs/EQ5D-5L-User-Guide.pdf. 2015. [Accessed May 9th, 2017].

51. Hsu E. Spirit (shen), styles of knowing, and authority in contemporary Chinese medicine. Culture, Medicine and Psychiatry. 2000;24(2):197-229.

52. Wong W, Lam CLK, Leung KF, Zhao L. Is the content of the Chinese Quality of Life Instrument (ChQOL) really valid in the context of traditional Chinese medicine in Hong Kong? Complementary Therapies in Medicine. 2009;17(1):29-36.

53. Liu F, Fang J. Chinese medicine and health-related quality of life. In: J F, ed. Health-related quality of life measurement and application. Beijing: Beijing Medical University Press; 2000:33-47.

54. Rossi E. Shen: psycho-emotional aspects of Chinese medicine. Edinburgh: Elsevier Health Sciences; 2007.

55. Zi T. Illustrating the fundamentals of the Yellow Emperor's Classic of Internal Medicine. Shaanxi: Shaanxi Normal University Press; 2012.

56. Deng T, Guo Z. Diagnostics in Chinese Medicine. Shanghai: Shanghai Science and Technology Publisher; 1984.

57. Hesketh T, Zhu WX. Health in China: Traditional Chinese medicine: one country, two systems. BMJ. 1997;315(7100):115-117.

58. $\mathrm{Xu} \mathrm{J}$, Yang Y. Traditional Chinese medicine in the Chinese health care system. Health policy. 2009;90(2-3):133-139.

59. Zhang H, Zhang F-y, Zhao Y-n, Li Z. Health concepts under the view of Chinese culture. Chinese Journal of Nursing. 2015;50(10):1236-1239.

60. Giacaman R, Khatib R, Shabaneh L, et al. What is health? The ability to adapt. The Lancet. 2009;373(9666):781-781. 
61. Huber M, Knottnerus JA, Green L, et al. How should we define health? BMJ. 2011;343:d4163.

62. Charlier P, Coppens Y, Malaurie J, et al. A new definition of health? An open letter of autochthonous peoples and medical anthropologists to the WHO. European Journal of Internal Medicine. 2017;37:33-37.

63. Wang G, Zhang J, Xu Y, Zhu Y, Ke D, Zhang G. Elementary study of sleep quality and self-rated health on common populations. Chinese Journal of Behavioral Medical Sciences. 2003;12(1):80-82.

64. Zhao J, Xie Y, Shi M, Ren Z, Feng X. Relationship between sleep quality and quality of life in military personnel in high altitude area. Chinese Journal of Public Health. 2006;22(6):665-666.

65. Cheng G, Zhang X, Yang Y. Study of Sleep and Quality of Life of the Workers from Chengdu Air Craft Manufacturing Factory. Modern Preventive Medicine 2008;35(1):45-47.

66. Lou C, Pang Z, Cui J. Practical Significance of Health Preservation Thoughts in Huangdi's Orthodox Classic. Li Shizhen Medicine and Medical Research. 2009;20(11):2837-2839.

67. Zhou X, Yu C, Wang H, et al. The relationship between Chinese traditional theory of essence-Qi-spirit and Health. Journal of Tianjin University of Traditional Chinese Medicine. 2013;32(1):8-11.

68. Zhao R. Theoretical Foundation of Chinese Food Culture In: Chinese Food Culture Beijing, China: Higher Education Press; 2003:10-25.

69. Han G, Ji J. A discussion on Chinese "Eating" Culture from the Greeting "Have you eaten". Youth Literator. 2009(5):76-77. 


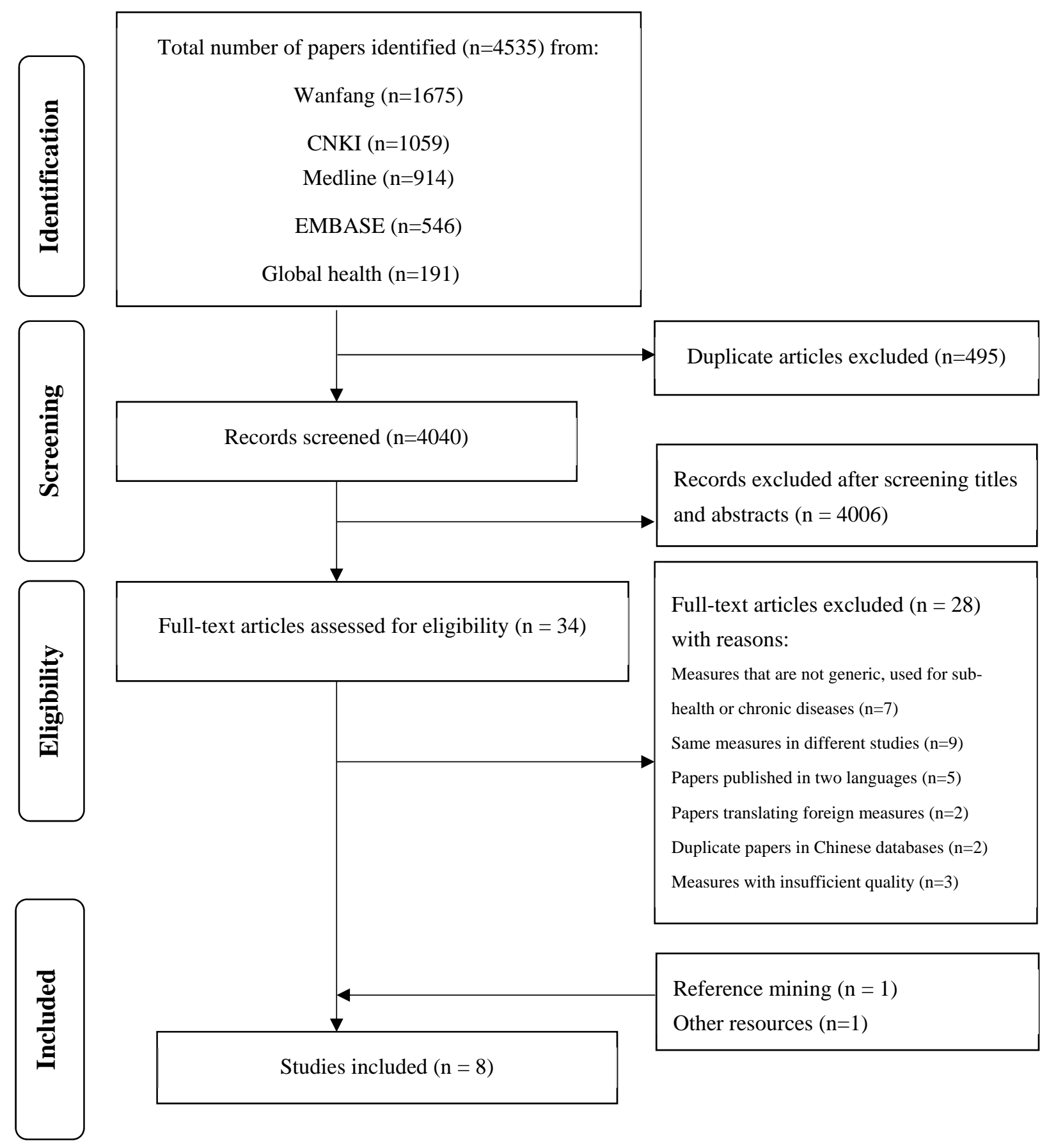

Fig.1 The process of study selection 


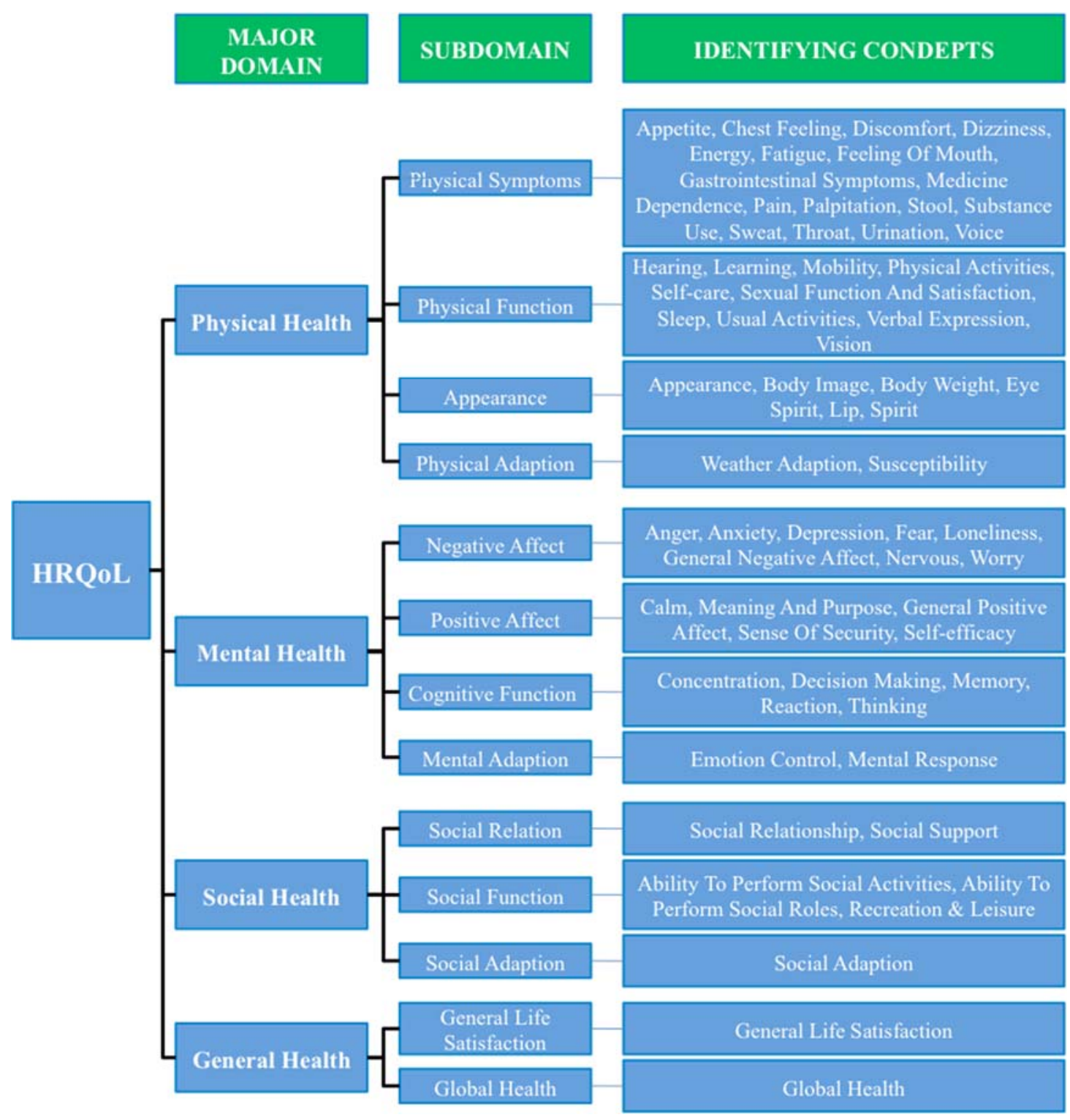

Fig 2. Working model of HRQoL domains, sub-domains, and concepts in the included measures 